\title{
Efficacy of Goal-Directed Fluid Therapy Monitored by Pulse-Pressure Variation Using a C ontinuous Non-Invasive Arterial Pressure Monitoring System (the CNAPTM System) During Parathyroidectomy in Patients with End-Stage Renal Failure - A randomized trial
}

Jie Song ( $1601905832 @ q q . c o m)$

Second Affiliated Hospital of Anhui Medical University https://orcid.org/0000-0001-8241-3447

Xiaofen Liu

Second Affiliated Hospital of Anhui Medical University

Weiwei Jiang

Second Affiliated Hospital of Anhui Medical University

Jiayou Wang

Second Affiliated Hospital of Anhui Medical University

Yun Li

Second Affiliated Hospital of Anhui Medical University

Hong Chen

Second Affiliated Hospital of Anhui Medical University

Ye Zhang

Second Affiliated Hospital of Anhui Medical University

Research article

Keywords: Goal-directed fluid therapy; Hemodynamic; Non-invasive monitoring; Pulse pressure variation; Anesthesia.

Posted Date: September 26th, 2019

DOI: https://doi.org/10.21203/rs.2.10803/v2

License: (c) (1) This work is licensed under a Creative Commons Attribution 4.0 International License. Read Full License 


\section{Abstract}

Background: There are no well-recognized guidelines for intraoperative fluid management in patients with end-stage renal failure (ESRF) . Goal-directed fluid therapy (GDFT) is a concept of perioperative fluid management that improves patients' prognosis. Dynamic indicators better predict fluid response than static indicators. Aim: In this study, we assessed a GDFT protocol with monitoring of pulse pressure variation (PPV) in patients with ESRF undergoing parathyroidectomy. Methods: The study included 102 patients who underwent elective parathyroidectomy. They were randomized to a control group (restrictive group, $n=51$ ) that was managed with a restricted fluid regimen or a PPV group (GDFT group, $n=51$ ) that was given a normal saline infusion and was monitored for change in PPV during the intraoperative period. If PPV reached $>13 \%, 250 \mathrm{~mL}$ normal saline was administered over $15 \mathrm{~min}$. Ephedrine was given at increments of $6 \mathrm{mg}$ to keep mean arterial pressure $>65 \mathrm{mmHg}$. Hemodynamic variables in the perioperative period were recorded. The primary endpoint was the occurrence of postoperative hypotension. Results: The occurrence of postoperative hypotension in the GDFT group was lower than in the restrictive group ( 0 vs. $11.67 \%, P=0.027$ ). The patients with complications in the GDFT group was lower than in the restrictive group $(35.3 \%$ vs. $54.9 \%, P=0.047)$. The volume of saline infused during the intraoperative period was 364 (219-408) $\mathrm{mL}$ in the GDFT group and 50 (50-50) $\mathrm{mL}$ in the restrictive group ( $P=0.001$ ). Ephedrine was given to $16 / 51(29.4 \%)$ of the GDFT group and $27 / 51(52.9 \%)$ of the restrictive group $(P=0.027)$. Conclusion: The use of goal-directed fluid therapy with the dynamic PPV indicator in patients with ESRF undergoing parathyroidectomy guides the administration of infused fluids, with reduced incidence of postoperative hypotension.

\section{Background}

There are no guidelines concerning perioperative fluid therapy for patients with end-stage renal failure (ESRF) [1]. Nearly all the studies of perioperative fluid therapy excluded patients with ESRF because of their physical condition [1-10].

Vascular diseases such as atherosclerosis predispose patients with secondary hyperparathyroidism (SHPT) to dramatic fluctuation of blood pressure in the perioperative period [11, 12]. Hypotension may be caused by restriction of intravenous fluid therapy and by the inhibitory effects of the anesthesia drugs on circulation, which could aggravate hypotension [11]. The administration of propofol and remifentanil may accentuate these problems [13]. Rational administration of drugs may help avert hypotension, but this measure may be insufficient [13].

For patients with SHPT, infusion volumes, which are based mainly on preoperative solute loads, should be carefully monitored [14]. To assess fluid status, noninvasive hemodynamic monitoring, as recorded with a continuous non-invasive arterial pressure monitoring system (CNAP) may be used [14]. CNAP can improve the control of blood pressure during dialysis, with resultant reduction in hospitalizations and without patient discomfort or vascular injury [15]. 
Goal-directed fluid therapy (GDFT), based on changes in stroke volume (SV) and cardiac index (Cl), has attracted much attention recently $[16,17]$. GDFT optimizes hemodynamics and oxygen delivery $[16,17]$. Monitoring pulse-pressure variation (PPV) may be more accurate than monitoring cardiac preload in patients on mechanical ventilation $[16,17]$. Many experts recommend that GDFT with PPV be used for all operations [18]. Pulse pressure (PP) is defined as the difference between systolic pressure and diastolic arterial pressure [19]. PPV is the variation in PP (Psystolic-Pdiastolic) caused by inspiration and expiration. Several factors can affect the accuracy of PPV, such as arrhythmia, spontaneous breathing, and peripheral vascular resistance [19]. No studies confirmed the impact of pathological changes in ESRF patients, including changes in increased pulmonary capillary permeability, calcification abnormalities, and cardiovascular dysfunction on the use of PPV.

GDFT, which can facilitate fluid management according to individual demographics and medical status, may be useful for such patients. Therefore, the purpose of this study was to determine the intraoperative fluid volume given to patients with SPTH and ESRF undergoing parathyroidectomy. We aimed to determine the safety and effectiveness of PPV in patients with ESRF by observing the occurrence of postoperative hypotension and optimize the infusion strategy by dynamic fluid management with CNAPPPV.

\section{Methods}

\section{Patients population}

We recruited 105 ESRF patients with SHPT who were scheduled for parathyroidectomy at our hospital between August and December 2018. Patients with primary hyperparathyroidism, severe pulmonary hypertension, arrhythmia, atherosclerosis, aortic stenosis, or chronic cardiac dysfunction were excluded. Patients with upper limb edema or malformation, or with blood pressure difference $>10 \mathrm{~mm} \mathrm{Hg}$ between the arms, were excluded. In total, three patients were excluded from this study. All patients were receiving hemodialysis thrice weekly or daily peritoneal dialysis. The patients had an American Society of Anesthesiologists physical status of III. Patients were randomized into two equal groups by a computerized random-number generator (managed by a third-party statistician): a control group, managed with a restricted-fluid regimen (restrictive group), and a PPV group (GDFT group) that was given normal saline infusion and monitored for change in PPV (Figure 1). The same operative team performed all operations.

\section{Anesthesia and mechanical ventilation}

No sedative or analgesic drugs were administered before the induction of anesthesia. Dialytic therapy was performed on the day before surgery. After their arrival in the operating room, patients received routine monitoring, including pulse oximetry $\left(\mathrm{SPO}_{2}\right)$, electrocardiogram, bispectral index (MedTronic, Minneapolis, MN, USA), and end-tidal $\mathrm{CO}_{2}$. CNAP was established and calibrated to measure blood 
pressure and other hemodynamic variables. We used the CNAP ${ }^{\mathrm{TM}}$ system (CNSystem, Medizintechnik, Graz, Austria).

General anesthesia was induced in all patients with bolus infusion and a target-controlled infusion of propofol (Fresenius Kabi AB, Macclesfield, UK) for a plasma concentration of 3.0-3.5 $\mu \mathrm{g} / \mathrm{mL}$; bolus of remifentanil (Yichang Humanwell Pharmaceutical Co., Ltd., Yichang, China) $1.5 \mu \mathrm{g} / \mathrm{kg}$ infused over $30 \mathrm{~s}$; and cisatracurium besylate $0.15 \mathrm{mg} / \mathrm{kg}$ (Jiangsu Hengrui Medicine Co., Ltd., Jiangsu, China). After tracheal intubation, ventilation was established with $6-8 \mathrm{~mL} / \mathrm{kg}$ tidal volume, and respiratory rate was adjusted to target end-tidal $\mathrm{CO}_{2}$ of $35-45 \mathrm{mmHg}$. Since the operation was performed and completed under endoscopy and in order to avoid excessive high airway pressure during the operation, we maintained the tidal volume at $8 \mathrm{ml} / \mathrm{kg}$, which was lowered (but still $>6 \mathrm{ml} / \mathrm{kg}$ ) only when the airway pressure was too high. Since the patient's PaCO2 had to remain within $35-45 \mathrm{mmHg}$, it did not achieve the criteria of permissive hypercapnia. Anesthesia was maintained with target-controlled infusion propofol (target concentration: 2.5-3.5 $\mu \mathrm{g} / \mathrm{ml})$, IV remifentanil $(0.2-0.3 \mu \mathrm{g} / \mathrm{kg} / \mathrm{min})$, and cisatracurium besylate $(0.05 \mathrm{mg} / \mathrm{kg} / \mathrm{min}$, intermittent intravenous injection). The intermittent injection of cisatracurium was based on the patients' muscle tone during the operation. During the operation, bispectral index values (MedTronic, Minneapolis, MN, USA) were maintained within $45 \pm 5$ by regulating the infusion rate of propofol. Thirty minutes before the end of the surgery, the cisatracurium besylate infusion was stopped. Propofol and remifentanil were turned off in both groups after wound closure.

The endotracheal tube was removed when patients were able to follow verbal commands to open their eyes, after checking for spontaneous respiration, swallowing, fist boxing, and keeping the head up before extubation, and the T7/T4 ratio was $90 \%$. Patients were kept in the post-anesthesia care unit for one hour.

\section{Fluid management}

In the restrictive group, only vasoactive agents were administered, without fluid infusion. In the GDFT group, intravenous fluid therapy and the use of vasoactive agents were determined according to the change of PPV and other hemodynamic variables. If PPV was $>13 \%, 250 \mathrm{~mL}$ of normal saline was administered over 15 min. Fluid responsiveness was evaluated every 15 min.

\section{Study parameters}

In both patient groups, demographic data, dialysis history, preoperative complications, duration of operation, total volume of anesthetics (propofol, remifentanil, and cisatracurium besylate) used, and intraoperative fluid and vasoactive agents infused were recorded. Postoperative complications, including hypotension, hypertension, pulmonary edema, infection, incision disunion, and arteriovenous fistula occlusionwere recorded. 
Vital signs and weight were recorded before and after the last dialysis and before the administration of anesthesia. Baseline SBP was the SBP measured after the last dialysis before surgery. The measurements were taken in the hemodialysis ward before transfer to the operating room. The SBP was measured in the supine position. Values were considered "maximum," "minimum," or "baseline." Hemodynamic variables were continually recorded at baseline (T0); before induction (T1); after induction (T2); immediately after intubation (T3); beginning of mechanical ventilation (T4); before incision (T5); 30 min, 60 min, and 90 min during the operation $(T 6, T 7, T 8)$; and 120 min during the operation or at the end of the operation if the operative time was $>120 \mathrm{~min}(\mathrm{~T} 9)$.

Blood samples were taken from the femoral artery before and 30 min after the operation and analyzed for brain natriuretic peptide, blood gases, hemoglobin/hematocrit, lactate, and electrolytes.

In order to preserve the integrity of the arteriovenous fistula, we avoided any blood pressure monitoring and puncture in the arm with the fistula. We used the lower extremity venous access, placed the arm with the arteriovenous fistula on the side of the body, and the nurse repeatedly confirmed that there was no compression of the venous fistula. Any abnormality of the arteriovenous fistula pulsation was assessed before and after the operation, in order to ensure functional integrity.

The primary endpoint of this study was hypotension after operation. The secondary endpoints were total volume of fluid administered, the doses of vasopressors used, the incidence of postoperative complications, abnormalities in blood gas values, and electrolyte concentrations.

Regarding complications, hypotension was considered when blood pressure is lower than the baseline blood pressure by $20 \%$. Hypertension was considered when blood pressure is higher than basic blood pressure by $20 \%$. Pulmonary edema was considered in the presence of hypoxemia, foamy sputum, auscultation of double lung wet rales, and confirmation by chest X-ray. Infections were confirmed by elevated C-reactive protein levels. Poor wound healing was defined as incision edema.

\section{Data and statistical analysis}

According to the records of the research center, postoperative hypotension rarely occurs in patients receiving dynamic fluid replacement, while postoperative hypotension is prone to occur with conventional surgery. Therefore, the difference in postoperative complications between the two groups was estimated at about $20 \%$. Postoperative hypotension was also considered in the calculation process of the minimum sample size. A sample of 44 patients in each group was required to detect a $20 \%$ reduction in postoperative hypotension at a significance level of 0.05 and power of $80 \%$. Considering a possible $20 \%$ dropout rate, a sample with a minimum of 53 per group was required.

Statistical analyses were performed with SPSS version 21.0 (IBM Corp. Released 2012. IBM SPSS Statistics for Windows, Version 21.0. Armonk, NY: IBM Corp). The data were tested for normal distribution with the Kolmogorov-Smirnov test and for homogeneity of variances with the Levene test [20]. Normally 
distributed continuous data were presented as mean \pm standard deviation, and those with abnormal distributions were expressed as median (25-75th percentile). Categorical variables were expressed as number (\%). The independent-samples $t$ test was used to compare continuous variables between the two groups, and repeated-measures one-way ANOVA was used for within-group comparisons. All enumeration data were compared using the chi-squared test or Fisher's exact test. Comparisons between ranked data were made with the Kruskal-Wallis test or the Wilcoxon test.

\section{Ethics}

The study was a single-blind randomized controlled trial, which was conducted at The Second Affiliated Hospital of Anhui Medical University, Hefei, China between August and December 2018. The protocol approved by the Ethics Committee for Clinical Trials of The Second Affiliated Hospital of Anhui Medical University (approval No. PJ-YX2018-008(F1)). Written informed consent was obtained from each patient. This trial was registered with the Chinese Clinical Trial Registry (ChiCTR1800017302). This manuscript adheres to the applicable Consolidated Standards of Reporting Trials (CONSORT) guidelines.

\section{Results}

\section{Patient characteristics}

One hundred and five patients with ESRF and SHPT undergoing parathyroidectomy were initially recruited in the study between August and December 2018; three were excluded, as illustrated in Figure 2. Thus, 102 patients were randomized (51 in the restrictive group and 51 in the GDFT group) and completed the study. Patients in both groups had similar baseline characteristics and comorbidities (Table 1).

\section{Intraoperative profiles}

The patients received total parathyroidectomy and re-implantation of a small parathyroid fragment subcutaneously in the femoral area to maintain normal hormone levels. Their intraoperative profiles are listed in Table 1. The median duration of operation was similar in the two groups (GDFT group, $122 \pm$ $18.3 \mathrm{~min}$; restrictive group, $117 \pm 15.5 \mathrm{~min}$. Patients in the GDFT group received significantly more saline infusion (median $364 \mathrm{~mL}$, range 219-408 mL) than did patients in the restrictive group (median $50 \mathrm{~mL}$, range 50-50 $\mathrm{mL}$; the need for infusion of intravenous anesthetics) $(P=0.001)$. Patients in the restrictive group received more vasoactive drugs than did those in the GDFT group; $27 / 51$ (52.9\%) received ephedrine compared with $16 / 51(29.4 \%)(P=0.027)$. Three patients in the restrictive group $(5.9 \%)$ also required continuous intravenous infusion of dopamine (they received 10, 20, and $38 \mathrm{mg}$, respectively), whereas none in the GDFT group needed this medication. The total volume of anesthetics used was similar in the two groups. There was no significant difference in blood loss between the groups, and no patient required transfusion. 
Figure 3 illustrates the perioperative hemodynamic changes that occurred in the two patient populations undergoing parathyroidectomy. Hemodynamic variables had no significant difference at baseline(T0)or before induction (T1). Compared with the baseline values, changes in hemodynamic variables at the time of after induction(T2), immediately after intubation (T3) were mostly similar in the two groups . At the time of mechanical ventilation (T4), SBP was slightly but statistically significantly lower, but diastolic blood pressure (DBP), mean blood pressure (MBP), and heart rate (HR) were not. After initiation of mechanical ventilation, the PPV value was similar between the two groups $[12.6 \pm 6.0 \%$ vs. $11.6 \pm 7.3 \% ; P$ $=0.417$, but after three fluid challenges (T6), the PPV was lower in the GDFT group than in the restrictive group $(8.9 \pm 2.8 \%$ vs. $11.6 \pm 5.1 \% ; P<0.001)$. The PPV remained lower in the GDFT group than in the restrictive group through $120 \mathrm{~min}$ or until the end of the operation (T9) $(7.5 \pm 2.1 \%$ vs. $11.4 \pm 5.3 \%$; $P<$ 0.001). SBP, DBP, and MBP were significantly lower through much or all of the operative period (T5-T9 or T7) in both patient groups than at T0. Through periods T5-T9, SBP was significantly higher in the GDFT group than in the restrictive group, whereas HR was higher in the restrictive group than in the GDFT group; PPV was significantly higher in the restrictive group than in the GDFT group at these time points. $\mathrm{Cl}$ was significantly higher in the GDFT group than in the restrictive group at T6, T7, and T9.

\section{Postoperative complications}

As the primary endpoint, the occurrence of postoperative hypotension was lower in the GDFT group (0/51; $0 \%)$ than in the restrictive group $(6 / 51 ; 11.7 \%)(P=0.027)$. Postoperative hypertension in the GDFT group $(18 / 51 ; 35.3 \%)$ and the restrictive group $(17 / 51 ; 33.3 \%)$ did not reach statistical significance $(P=0.500)$. Arteriovenous fistula occlusion was lower in the GDFT group $(0 / 51 ; 0 \%)$ than in the restrictive group $(8 / 51 ; 15.7 \%)(P=0.006)$. Among those eight patients, one had preoperative diarrhea, two had a long history of hypotension (mean systolic blood pressure $70-80 \mathrm{mmHg}$ ), one had long operation time ( $>3 \mathrm{~h}$ ), one had preoperative damage of arteriovenous fistula, and one had diabetes-related vascular disease; the reason could not be identified in the remaining two patients. In addition, one patient suffered from myocardial infarction after surgery in the restrictive group. Although this was not a complication as per study design, this could be related to intraoperative hemodynamic changes. The patients with complications were less in the GDFT group $(18 / 51 ; 35.3 \%)$ than in the restrictive group $(28 / 51 ; 54.9 \%)$ $(P=0.047)$. No patient suffered from complications due to pulmonary edema, infection, or incision disunion (Table 2).

\section{Baseline and postoperative laboratory tests}

Baseline and postoperative values are presented in Table 3. A mild but statistically significant drop in hematocrit occurred in the GDFT group (from $39.5 \pm 5.5$ to $37.2 \pm 5.1, P=0.034$ ), whereas no drop occurred in the restrictive group. No significant differences in other laboratory tests were recorded. 


\section{Discussion}

Parathyroidectomy is the most frequently performed operation in ESRF patients [21], but there are no wellrecognized guidelines for intraoperative fluid management. In this study, we aimed to optimize fluid management in patients with SPTH and ESRF undergoing parathyroidectomy. Thus, we applied the CNAP system to guide GDFT during the perioperative period. These strategies provided fluid responsiveness to help regulate venous return and $\mathrm{Cl}$ to reduce the incidence of hypotension and subsequent adverse events. Previous GDFT studies used invasive monitors under mechanical ventilation [22, 23], whereas we used a noninvasive system. With our protocol, the hemodynamics were well maintained, the use of vasoconstrictive drugs was reduced, and the complications were fewer than in patients managed with conventional fluid administration. The protocol is feasible in the fluid management of hemodialysis patients.

Parathyroidectomy can delay the progression of the disorder and improve the quality of life for patients with SHPT [24]. Long-term hypertension and hypercalcemia in SHPT patients can accentuate their propensity to dramatic fluctuation of blood pressure in the perioperative period, especially after induction $[11,12]$. In this study, more patients in the restrictive group than in the GDFT group had hypotension after operation, as supported by a previous study that reported an occurrence of $19 \%$ [25]. Due to preoperative fasting, dialysis and non-urinary fluid loss, even patients with ESRF may have intraoperative hypovolemia $[11,12]$. Thus, restricted intravenous fluid therapy makes such patients susceptible to hypotension, which can be aggravated from anesthesia, especially when using propofol and remifentanil [13].

High blood viscosity, low blood volume, endovascular intima damage, thrombosis, and improper care, among others, are all possible reasons for arteriovenous fistula occlusion [26], which is a dismal complication because it can complicate future dialysis. Eight patients in our restrictive group had arteriovenous fistula occlusion compared with none in the GDFT group. It is consistent with evidence that vascular occlusion is one of the most serious complications due to hypotension and unstable blood pressure during surgery $[27,28]$. Thus, adequate volume expansion to maintain stable hemodynamics and perfusion is critical. Despite significant associations between the use of GDFT and the lower occurence rate of postoperative hypotension and arteriovenous fistula occlusion, the exact causal relationship remains to be determined.

Controversy exists over the strategy of fluid management of patients with SHPT during anesthesia [29, 30]. Some physicians choose no infusion because of fear of fluid overload. Since SHPT patients have a range of sensibility to vasoactive drugs, the incidence of hypertension and/or hypotension in them is high [31, 32]. In addition, some drugs used for anesthesia may reduce the oxygen supply to vital organs [33]. Thus, rational drug use is necessary but may not be enough to maintain normal hemodynamics. Therefore, some authors advocate individualized fluid management during the surgery for patients with SHPT $[34,35]$. Recent studies revealed associations between hypotension and adverse outcomes such as myocardial injury, depending on the extent and duration of hypotension [36-38]. In non-cardiac surgery, the most common cardiac complication is myocardial infarction, which may be caused by an imbalance 
between myocardial oxygen supply and demand [36-38].. A meta-analysis based on multiple cohort studies showed that intraoperative hypotension increased postoperative major cardiovascular events $(O R=1.56)$, especially for myocardial injury (OR=1.67) [37]. Salmasi et al. [38] found that MAP below the absolute value of $65 \mathrm{mmHg}$ or a decrease of $>20 \%$ of baseline can increase the risk of postoperative myocardial damage. The main goal of perioperative fluid management is optimal microcirculatory perfusion, which can be achieved with well-controlled blood pressure and adequate volume expansion [39]. Some authors suggest that the right amount of fluid can be input during parathyroidectomy, but the absolute amount of liquid should not be fixed [38]. If the amount of dehydration of the last hemodialysis $\geq 3 \mathrm{~kg}$, patients with normal cardiac function are often accompanied by different degrees of dehydration, but in patients with cardiac insufficiency, there is still the problem of extracellular fluid overload [38]. Therefore, if there is insufficient capacity before the start of anesthesia, the blood volume should be replenished in time, and the cardiac function should be considered. Otherwise, huge hemodynamic fluctuations will occur after anesthesia.

The infusion volume is mainly determined by the preoperative state of solute loads [40,41]. Thus, it is important to know the patients' actual weight and dry weight [41]. The dry weight is the lowest weight that can be safely attained after dialysis without hypotension developing [42]. Prolonged low diastolic pressure is one of the independent risk factors for cardiovascular complications [43]. The risk of postoperative pulmonary edema and hypertension is increased in patients whose weight is higher than their dry weight; this imbalance can impede wound healing and increase the chance of infection [44]. These complications caused by fluid under/over-load are the focus of our research.

To achieve and maintain dry weight, the use of noninvasive hemodynamic monitoring such as with a CNAP monitoring system to monitor the water load in hemodialysis has been advocated [45]. It first measures arterial blood pressure through the upper-arm calibration system and blood volume and pressure signal through double fingertip-sensors continuously. Then, using the vascular unload technique and VERIFI algorithm, it eliminates the contrast artifact [46]. CNAP can improve blood pressure control between dialysis sessions and limit hospitalizations [47]. In our study, the CNAP system provided consistent hemodynamic measurements without causing patient discomfort.

Nevertheless, the exact required volume of fluid expansion is difficult to predict and varies among individuals $[40,41]$. By providing individualized fluid management, GDFT may help solve this problem. A large PPV or an increase in PPV can be interpreted as operating on the steep portion of the Frank-Starling curve, warning the responsible physician to counteract further fluid depletion to avoid hemodynamic instability [48]. By monitoring non-invasive parameter PPV, this indicator could efficiently assess the fluid requirements of patients with general anesthesia and mechanical ventilation [49]. MAP, PPV, Cl, SVR, and other parameters should be considered comprehensively in patients under general anesthesia with mechanical ventilation in order to accurately assess liquid reactivity [50,51]. CNAP can provide real-time PPV monitoring, and CNAP-PPV has identical sensitivity and accuracy to that of invasive methods [52, 53]. 
In the present study, GDFT strategies via CNAP-PPV enabled fluid responsiveness to optimize venous return and $\mathrm{Cl}$ to reduce the occurrence of hypotension and subsequent adverse events. The GDFT strategies we used in our study reduced the total dosages of vasopressors administered, thus reducing the heart rate, which can be increased using ephedrine and dopamine. Furthermore, after moderate fluid expansion, hematocrit decreases, and hemoconcentration seems to be improved. As verified by our data, the excessive use of vasoconstriction drugs without adequate fluid loading may further induce vasoconstriction, which may cause serious complications after surgery, similar to arteriovenous fistula.

\section{Limitations of the study}

We acknowledge that this study has limitations. Since there is little bleeding and the operation time is short with parathyroidectomy, the results may not be applicable to major operations. Some hemodialysis patients have arteriovenous fistulae on both arms or severe arrhythmia, so our protocol will not apply to them. The dry weight of the patients and their weight gain after surgery were not recorded. Our anesthesia team only observed the condition of hemodialysis on the first day after surgery, and subsequent observation and treatment were not included in the study. This study did not observe a correlation between postoperative complications and the duration of surgery.

\section{Conclusions}

In the current study, we show that PPV-guided GDFT with the CNAP system during parathyroidectomy in ESRF patients is feasible and reliable. The GDFT protocol reported in this study maintained hemodynamic stability, reduced the requirements of vasopressors, and decreased postoperative adverse events.

\section{Declarations}

\section{Ethics approval and consent to participate}

All procedures performed in studies involving human participants were in accordance with the ethical standards of the institutional and/or national research committee and with the 1964 Helsinki declaration and its later amendments or comparable ethical standards. The protocol approved by the Ethics Committee for Clinical Trials of The Second Affiliated Hospital of Anhui Medical University (approval No. PJ-YX2018-008(F1)). Written informed consent was obtained from each patient. This trial was registered with the Chinese Clinical Trial Registry (ChiCTR1800017302). This manuscript adheres to the applicable Consolidated Standards of Reporting Trials (CONSORT) guidelines.

\section{Consent to publish}


There are no details on individuals reported within the manuscript.

\section{Availability of data and materials}

The datasets used and/or analysed during the current study are available from the corresponding author on reasonable request.

\section{Competing interests}

The authors declare that they have no competing interests.

\section{Funding}

This study is supported by the Priority Department of Second Affiliated Hospital of Anhui Medical University.

\section{Authors' Contributions}

Jie Song: This author helped design the study, conduct the study, analyze the data, and write and revise the paper.

Xiaofen Liu: This author helped analyze the data.

Weiwei Jiang: This author helped conduct the study.

Jiayou Wang: This author helped conduct the study.

Yun Li: This author helped design the study.

Hong Chen: This author helped conduct the study.

Ye Zhang: This author helped design the study and write and revise the paper.

\section{Author details}

Department of Anesthesiology, the Second Affiliated Hospital of Anhui Medical University, Anhui 230032, China 
Acknowledgments

The authors are grateful to the participating patients. We acknowledge Shengxue Xie, MM, Liquan YU, MM, and Peikun Li, MM, Department of General Surgery of the Second Affiliated Hospital of Anhui Medical University, for assistance of postoperative follow-up of patients. We acknowledge the expertise of Peng Zhu (School of Public Health, Anhui Medical University) in the revision of the manuscript, as well as Juan Zhou, Chun-xiao Wu, Xiao-yan Zhang and Qin Li for their excellent help with patient organization and nursing assistance.

\section{References}

1. Joosten A, Huynh T, Suehiro K, Canales C, Cannesson M, Rinehart J: Goal-Directed fluid therapy with closed-loop assistance during moderate risk surgery using noninvasive cardiac output monitoring: A pilot study. Br J Anaesth 2015, 114:886-892.

2. Miller TE, Roche AM, Mythen M: Fluid management and goal-directed therapy as an adjunct to Enhanced Recovery After Surgery (ERAS). Can J Anaesth 2015, 62:158-168.

3. Joosten A, Coeckelenbergh S, Delaporte A, Ickx B, Closset J, Roumeguere T, Barvais L, Van Obbergh L, Cannesson M, Rinehart J, Van der Linden P: Implementation of closed-loop-assisted intra-operative goal-directed fluid therapy during major abdominal surgery: A case-control study with propensity matching. Eur J Anaesthesio/ 2018, 35:650-658.

4. Joosten A, Delaporte A, Ickx B, Touihri K, Stany I, Barvais L, Van Obbergh L, Loi P, Rinehart J, Cannesson M, Van der Linden P: Crystalloid versus Colloid for Intraoperative Goal-directed Fluid Therapy Using a Closed-loop System: A Randomized, Double-blinded, Controlled Trial in Major Abdominal Surgery. Anesthesiology 2018, 128:55-66.

5. Joosten A, Hafiane R, Pustetto M, Van Obbergh L, Quackels T, Buggenhout A, Vincent JL, Ickx B, Rinehart J: Practical impact of a decision support for goal-directed fluid therapy on protocol adherence: a clinical implementation study in patients undergoing major abdominal surgery. $J$ Clin Monit Comput 2019, 33:15-24.

6. Joosten A, Raj Lawrence S, Colesnicenco A, Coeckelenbergh S, Vincent JL, Van der Linden P, Cannesson M, Rinehart J: Personalized Versus Protocolized Fluid Management Using Noninvasive Hemodynamic Monitoring (Clearsight System) in Patients Undergoing Moderate-Risk Abdominal Surgery. Anesth Analg 2019, 129:e8-e12.

7. Rinehart J, Lilot M, Lee C, Joosten A, Huynh T, Canales C, Imagawa D, Demirjian A, Cannesson M: Closed-loop assisted versus manual goal-directed fluid therapy during high-risk abdominal surgery: a case-control study with propensity matching. Crit Care 2015, 19:94.

8. Edwards MR, Forbes G, MacDonald N, Berdunov V, Mihaylova B, Dias P, Thomson A, Grocott MP, Mythen MG, Gillies MA, et al: Optimisation of Perioperative Cardiovascular Management to Improve Surgical Outcome II (OPTIMISE II) trial: study protocol for a multicentre international trial of cardiac 
output-guided fluid therapy with low-dose inotrope infusion compared with usual care in patients undergoing major elective gastrointestinal surgery. BMJ Open 2019, 9:e023455.

9. MacDonald N, Pearse RM: Are we close to the ideal intravenous fluid? Br J Anaesth 2017, 119:i63i71.

10. Miller TE, Pearse RM: Perioperative fluid management: moving toward more answers than questionsa commentary on the RELIEF study. Perioper Med (Lond) 2019, 8:2.

11. Ishani A, Liu J, Wetmore JB, Lowe KA, Do T, Bradbury BD, Block GA, Collins AJ: Clinical outcomes after parathyroidectomy in a nationwide cohort of patients on hemodialysis. Clin J Am Soc Nephrol 2015, 10:90-97.

12. London GM, Drueke TB: Atherosclerosis and arteriosclerosis in chronic renal failure. Kidney Int 1997, 51:1678-1695.

13. Mazzuchi N, Carbonell E, Fernandez-Cean J: Importance of blood pressure control in hemodialysis patient survival. Kidney Int 2000, 58:2147-2154.

14. Santos JF, Parreira L, Madeira J, Fonseca N, Soares LN, Ines L: Non invasive hemodynamic monitorization for AV interval optimization in patients with ventricular resynchronization therapy. Rev Port Cardiol 2003, 22:1091-1098.

15. Lindberg M, Prutz KG, Lindberg P, Wikstrom B: Interdialytic weight gain and ultrafiltration rate in hemodialysis: lessons about fluid adherence from a national registry of clinical practice. Hemodial Int 2009, 13:181-188.

16. Rollins KE, Lobo DN: Intraoperative Goal-directed Fluid Therapy in Elective Major Abdominal Surgery: A Meta-analysis of Randomized Controlled Trials. Ann Surg 2016, 263:465-476.

17. Scheeren TW, Wiesenack C, Gerlach H, Marx G: Goal-directed intraoperative fluid therapy guided by stroke volume and its variation in high-risk surgical patients: a prospective randomized multicentre study. J Clin Monit Comput 2013, 27:225-233.

18. Salzwedel C, Puig J, Carstens A, Bein B, Molnar Z, Kiss K, Hussain A, Belda J, Kirov MY, Sakka SG, Reuter DA: Perioperative goal-directed hemodynamic therapy based on radial arterial pulse pressure variation and continuous cardiac index trending reduces postoperative complications after major abdominal surgery: a multi-center, prospective, randomized study. Crit Care 2013, 17:R191.

19. Daudel F, Tuller D, Krahenbuhl S, Jakob SM, Takala J: Pulse pressure variation and volume responsiveness during acutely increased pulmonary artery pressure: an experimental study. Crit Care 2010, 14:R122.

20. Nazemi M, Raad MH, Arzoomanian CS, Ghasemzadeh A: Fatigue and Depression in Iranian Amyotrophic Lateral Sclerosis Patients in Tehran in 2012. Electron Physician 2016, 8:2194-2198.

21. Oltmann SC, Madkhali TM, Sippel RS, Chen H, Schneider DF: Kidney Disease Improving Global Outcomes guidelines and parathyroidectomy for renal hyperparathyroidism. J Surg Res 2015, 199:115-120.

22. Marik PE: Noninvasive cardiac output monitors: a state-of the-art review. J Cardiothorac Vasc Anesth 2013, 27:121-134. 
23. Vincent JL, Rhodes A, Perel A, Martin GS, Della Rocca G, Vallet B, Pinsky MR, Hofer CK, Teboul JL, de Boode WP, et al: Clinical review: Update on hemodynamic monitoring-a consensus of 16. Crit Care 2011, 15:229.

24. Yuen NK, Ananthakrishnan S, Campbell MJ: Hyperparathyroidism of Renal Disease. Perm J 2016, 20:15-127.

25. Corneci M, Stanescu B, Trifanescu R, Neacsu E, Corneci D, Poiana C, Horvat T: Perioperative management difficulties in parathyroidectomy for primary versus secondary and tertiary hyperparathyroidism. Maedica (Buchar) 2012, 7:117-124.

26. Smith GE, Gohil R, Chetter IC: Factors affecting the patency of arteriovenous fistulas for dialysis access. J Vasc Surg 2012, 55:849-855.

27. Khan A, Khan AH, Adnan AS, Syed Sulaiman SA, Gan SH, Khan I: Management of Patient Care in Hemodialysis While Focusing on Cardiovascular Disease Events and the Atypical Role of Hyperand/or Hypotension: A Systematic Review. Biomed Res Int 2016, 2016:9710965.

28. Palevsky PM: Perioperative management of patients with chronic kidney disease or ESRD. Best Pract Res Clin Anaesthesiol 2004, 18:129-144.

29. Doherty M, Buggy DJ: Intraoperative fluids: how much is too much? Br J Anaesth 2012, 109:69-79.

30. Minto G, Mythen MG: Perioperative fluid management: science, art or random chaos? Br J Anaesth 2015, 114:717-721.

31. Ackland GL, Moran N, Cone S, Grocott MP, Mythen MG: Chronic kidney disease and postoperative morbidity after elective orthopedic surgery. Anesth Analg 2011, 112:1375-1381.

32. Zager PG, Nikolic J, Brown RH, Campbell MA, Hunt WC, Peterson D, Van Stone J, Levey A, Meyer KB, Klag MJ, et al: "U" curve association of blood pressure and mortality in hemodialysis patients. Medical Directors of Dialysis Clinic, Inc. Kidney Int 1998, 54:561-569.

33. Nissen P, Brassard P, Jorgensen TB, Secher NH: Phenylephrine but not ephedrine reduces frontal lobe oxygenation following anesthesia-induced hypotension. Neurocrit Care 2010, 12:17-23.

34. Baxi V, Jain A, Dasgupta D: Anaesthesia for renal transplantation: an update. Indian J Anaesth 2009, 53:139-147.

35. Meersch M, Schmidt C, Zarbock A: Patient with chronic renal failure undergoing surgery. Curr Opin Anaesthesiol 2016, 29:413-420.

36. Sessler DI, Khanna AK: Perioperative myocardial injury and the contribution of hypotension. Intensive Care Med 2018, 44:811-822.

37. van Waes JA, van Klei WA, Wijeysundera DN, van Wolfswinkel L, Lindsay TF, Beattie WS: Association between Intraoperative Hypotension and Myocardial Injury after Vascular Surgery. Anesthesiology 2016, 124:35-44.

38. Salmasi V, Maheshwari K, Yang D, Mascha EJ, Singh A, Sessler DI, Kurz A: Relationship between Intraoperative Hypotension, Defined by Either Reduction from Baseline or Absolute Thresholds, and 
Acute Kidney and Myocardial Injury after Noncardiac Surgery: A Retrospective Cohort Analysis. Anesthesiology 2017, 126:47-65.

39. Bennett VA, Cecconi M: Perioperative fluid management: From physiology to improving clinical outcomes. Indian J Anaesth 2017, 61:614-621.

40. Voldby AW, Brandstrup B: Fluid therapy in the perioperative setting-a clinical review. J Intensive Care 2016, 4:27.

41. Craig RG, Hunter JM: Recent developments in the perioperative management of adult patients with chronic kidney disease. Br J Anaesth 2008, 101:296-310.

42. Charra B, Chazot C: Volume control, blood pressure and cardiovascular function. Lessons from hemodialysis treatment. Nephron Physio/ 2003, 93:p94-101.

43. Peralta CA, Shlipak MG, Wassel-Fyr C, Bosworth H, Hoffman B, Martins S, Oddone E, Goldstein MK: Association of antihypertensive therapy and diastolic hypotension in chronic kidney disease. Hypertension 2007, 50:474-480.

44. Kanda H, Hirasaki Y, lida T, Kanao-Kanda M, Toyama Y, Chiba T, Kunisawa T: Perioperative Management of Patients With End-Stage Renal Disease. J Cardiothorac Vasc Anesth 2017, 31:22512267.

45. Wimmer J, Batzel JJ, Haditsch B, Schneditz D: Evolution of volume sensitivity during hemodialysis and ultrafiltration. Clin Auton Res 2011, 21:353-360.

46. Jeleazcov C, Krajinovic L, Munster T, Birkholz T, Fried R, Schuttler J, Fechner J: Precision and accuracy of a new device (CNAPTM) for continuous non-invasive arterial pressure monitoring: assessment during general anaesthesia. Br J Anaesth 2010, 105:264-272.

47. Yoshii M, Minami J, Ishimitsu T, Yamakoshi K, Matsuoka H: Non-invasive monitoring of hemodynamic changes during hemodialysis by the use of a newly developed admittance cardiograph. Ther Apher Dial 2005, 9:154-160.

48. Michard F: Changes in arterial pressure during mechanical ventilation. Anesthesiology 2005, 103:419-428; quiz 449-415.

49. Monnet X, Dres M, Ferre A, Le Teuff G, Jozwiak M, Bleibtreu A, Le Deley MC, Chemla D, Richard C, Teboul JL: Prediction of fluid responsiveness by a continuous non-invasive assessment of arterial pressure in critically ill patients: comparison with four other dynamic indices. Br J Anaesth 2012, 109:330-338.

50. Marik PE: Fluid Responsiveness and the Six Guiding Principles of Fluid Resuscitation. Crit Care Med 2016, 44:1920-1922.

51. Carsetti A, Cecconi M, Rhodes A: Fluid bolus therapy: monitoring and predicting fluid responsiveness. Curr Opin Crit Care 2015, 21:388-394.

52. Biais M, Stecken L, Ottolenghi L, Roullet S, Quinart A, Masson F, Sztark F: The ability of pulse pressure variations obtained with CNAP device to predict fluid responsiveness in the operating room. Anesth Analg 2011, 113:523-528. 
53. Biais M, Stecken L, Martin A, Roullet S, Quinart A, Sztark F: Automated, continuous and non-invasive assessment of pulse pressure variations using $\operatorname{CNAP((R))~system.~J~Clin~Monit~Comput~2017,~}$ 31:685-692.

\section{Tables}

Table 1. Patient Characteristics and Preoperative Profiles

\begin{tabular}{|c|c|c|c|}
\hline Characteristic & $\begin{array}{l}\text { GDFT group }(n= \\
51)\end{array}$ & $\begin{array}{l}\text { Restrictive group ( } \mathrm{n}= \\
51 \text { ) }\end{array}$ & $P$ \\
\hline $\operatorname{Sex}(M / F)$ & $36 / 15$ & $32 / 19$ & 0.401 \\
\hline Age (years) & $47.49 \pm 7.56$ & $46.59 \pm 6.65$ & 0.524 \\
\hline Height (cm) & $163.47 \pm 7.62$ & $165.49 \pm 8.13$ & 0.199 \\
\hline Weight (kg) & $58.92 \pm 10.52$ & $62.73 \pm 12.1$ & 0.093 \\
\hline BMI $\left(\mathrm{kg} / \mathrm{m}^{2}\right)$ & $22.01 \pm 3.21$ & $23.44 \pm 5.44$ & 0.108 \\
\hline History of dialysis (years) & $7.55 \pm 3.16$ & $7.73 \pm 2.32$ & 0.749 \\
\hline \multicolumn{4}{|l|}{ Comorbidities } \\
\hline Hypertension & $38(74.5 \%)$ & $36(70.6 \%)$ & 0.657 \\
\hline $\begin{array}{l}\text { Cardiac disease (except } \\
\text { hypertension) }\end{array}$ & $8(15.7 \%)$ & $5(9.8 \%)$ & 0.373 \\
\hline Pulmonary disease & $14(27.5 \%)$ & $17(33.3 \%)$ & 0.518 \\
\hline Diabetes mellitus & $13(25.5 \%)$ & $10(19.6 \%)$ & 0.477 \\
\hline Gastrointestinal disease & 19 (37.3\%) & $20(39.2 \%)$ & 0.839 \\
\hline Anemia & 39 (76.5\%) & $34(66.7 \%)$ & 0.272 \\
\hline Fasting duration (hours) & $10.2 \pm 2.1$ & $10.6 \pm 2.8$ & 0.416 \\
\hline Systolic blood pressure & $142 \pm 25$ & $140 \pm 26$ & 0.745 \\
\hline Diastolic blood pressure & $79 \pm 13$ & $79 \pm 16$ & 0.931 \\
\hline Mean blood pressure & $99.7 \pm 16.0$ & $99.4 \pm 18.2$ & 0.926 \\
\hline Heart rate & $73 \pm 9$ & $74 \pm 9$ & 0.667 \\
\hline
\end{tabular}

Values are given as mean value $\pm \mathrm{SD}$, median (25-75th percentile), or percentages. 
Table 2. Intraoperative Profiles

\begin{tabular}{llll}
\hline Characteristic & GDFT group $(\mathrm{n}=$ & Restrictive group $(\mathrm{n}=$ & $P$ \\
& 51) & 51) & \\
\hline Operation time (min) & $122 \pm 18$ & $117 \pm 15$ & 0.126 \\
Total volume of normal saline & $363.7(219.1-407.9)$ & $50.0(50-50)$ & 0.001
\end{tabular}

(mL)

Stroke volume (mL)

\begin{tabular}{clll} 
T1 & $68.73 \pm 10.88$ & $68.01 \pm 11.07$ & 0.741 \\
T2 & $66.74 \pm 11.35$ & $67.91 \pm 11.98$ & 0.614 \\
T3 & $68.76 \pm 10.67$ & $69.56 \pm 11.22$ & 0.713 \\
\hline T4 & $65.39 \pm 10.90$ & $67.39 \pm 15.43$ & 0.452 \\
T5 & $68.18 \pm 12.87$ & $69.29 \pm 12.81$ & 0.661 \\
\hline T6 & $67.29 \pm 15.60$ & $74.51 \pm 20.51$ & 0.048 \\
\hline T7 & $68.63 \pm 13.73$ & $73.73 \pm 19.40$ & 0.129 \\
T8 & $67.63 \pm 13.70$ & $72.90 \pm 18.87$ & 0.109 \\
\hline T9 & $69.90 \pm 14.710$ & $73.24 \pm 18.92$ & 0.323
\end{tabular}

Frequency of vasoactive drugs given

$\begin{array}{lllr}\text { Ephedrine } & 16(29.4 \%) & 27(52.9 \%) & 0.027 \\ \text { Dopamine } & 0 & 3(5.9 \%) & 0.241\end{array}$

Total volume of vasoactive drugs

\begin{tabular}{llll} 
Ephedrine $(\mathrm{mg})$ & $0(0-12)$ & $12(0-24)$ & 0.008 \\
\hline Dopamine $(\mathrm{mg})$ & 0 & $20(10-38)$ & 0.609
\end{tabular}

Total volume of anesthetics

\begin{tabular}{llll} 
Propofol $(\mathrm{mg})$ & $438.00 \pm 68.00$ & $441.00 \pm 78.00$ & 0.836 \\
\hline Remifentanil $(\mathrm{mg})$ & $2.10 \pm 1.20$ & $2.40 \pm 0.80$ & 0.141 \\
\hline Cisatracurium besylate $(\mathrm{mg})$ & $9.80 \pm 2.10$ & $10.20 \pm 1.70$ & 0.292 \\
\hline
\end{tabular}


Values are given as mean value $\pm \mathrm{SD}$, median (25-75th percentile), median (range) or percentages

Table 3. Postoperative Complications

GDFT group Restrictive group $P$

\begin{tabular}{llll} 
& $(\mathbf{n}=\mathbf{5 1 )}$ & $\mathbf{( n = 5 1 )}$ & \\
\hline Hypotension & 0 & $6(11.8 \%)$ & 0.027 \\
\hline Hypertension & $18(35.3 \%)$ & $17(33.3 \%)$ & 0.500 \\
\hline Arteriovenous fistula occlusion & 0 & $8(15.7 \%)$ & 0.006 \\
\hline Others & 0 & $1(2.0 \%)$ & 1.000 \\
\hline
\end{tabular}


Table 4. Baseline and Postoperative Laboratory Tests

\begin{tabular}{|c|c|c|c|}
\hline Parameter & GDFT group & Restrictive group & $P$ \\
\hline \multicolumn{4}{|l|}{$\mathrm{pH}$} \\
\hline Baseline & $7.41 \pm 0.07$ & $7.4 \pm 0.05$ & 0.128 \\
\hline Post-operation & $7.36 \pm 0.05$ & $7.37 \pm 0.05$ & 0.560 \\
\hline \multicolumn{4}{|l|}{$\mathrm{PaO}_{2}(\mathrm{mmHg})$} \\
\hline Baseline & $466.25 \pm 54.41$ & $463.73 \pm 56.03$ & 0.818 \\
\hline Post-operation & $456.47 \pm 40.37$ & $462.24 \pm 46.25$ & 0.504 \\
\hline \multicolumn{4}{|l|}{$\mathrm{PaCO}_{2}(\mathrm{mmHg})$} \\
\hline Baseline & $43.33 \pm 5.52$ & $43.94 \pm 4.46$ & 0.541 \\
\hline Post-operation & $44.98 \pm 5.81$ & $45.8 \pm 5.84$ & 0.477 \\
\hline \multicolumn{4}{|l|}{$\mathrm{HCO}_{3}^{-}(\mathrm{mmol} / \mathrm{L})$} \\
\hline Baseline & $25.99 \pm 2.57$ & $26.09 \pm 2.06$ & 0.822 \\
\hline Post-operation & $25.86 \pm 2.46$ & $25.63 \pm 2.07$ & 0.608 \\
\hline
\end{tabular}

Hematocrit (mm)

Baseline

$39.12 \pm 5.35$

$39.35 \pm 5.25$

0.823

Post-operation

$37.24 \pm 5.14$

$39.49 \pm 5.47$

0.034

Hemoglobin ( $\mathrm{g} / \mathrm{L}$ )

Baseline

$97.20 \pm 13.40$

$98.20 \pm 11.10$

0.682

Post-operation

$96.30 \pm 12.20$

$97.70 \pm 12.40$

0.567

Lactate $(\mathrm{mmol} / \mathrm{L})$

Baseline

$1.06 \pm 0.49$

$0.95 \pm 0.41$

0.188

Post-operation

$1.03 \pm 0.46$

$1.06 \pm 0.43$

0.774

BNP $(p g / m L)$

Baseline

$280.20 \pm 253.30 \quad 299.30 \pm 212.30 \quad 0.681$

Post-operation

$278.50 \pm 391.90$

$286.30 \pm 298.70$

0.910

Serum sodium ( $\mathrm{mmol} / \mathrm{L})$

Baseline

$137.18 \pm 2.21$

$137.61 \pm 2.45$

0.352

Post-operation

$136.22 \pm 3.64$

$137.10 \pm 2.61$

0.163 
Serum potassium $(\mathrm{mmol} / \mathrm{L})$

\begin{tabular}{cccc}
\hline Baseline & $4.21 \pm 0.50$ & $4.37 \pm 0.39$ & 0.071 \\
\hline Post-operation & $4.42 \pm 0.74$ & $4.48 \pm 0.62$ & 0.666 \\
\hline Serum calcium $(\mathrm{mmol} / \mathrm{L})$ & & & \\
\hline Baseline & $1.17 \pm 0.09$ & $1.17 \pm 0.12$ & 0.953 \\
\hline Post-operation & $1.16 \pm 0.1$ & $1.16 \pm 0.09$ & 1.000 \\
\hline Serum chloride $(\mathrm{mmol} / \mathrm{L})$ & & & \\
\hline Baseline & $101.30 \pm 2.10$ & $101.50 \pm 1.80$ & 0.607 \\
\hline Post-operation & $100.50 \pm 1.90$ & $100.90 \pm 1.40$ & 0.229 \\
\hline
\end{tabular}

BNP, brain natriuretic peptide. Values are given as mean value \pm SD. $P$ values in the table indicate the statistical significance between the two groups.

\section{Figures}

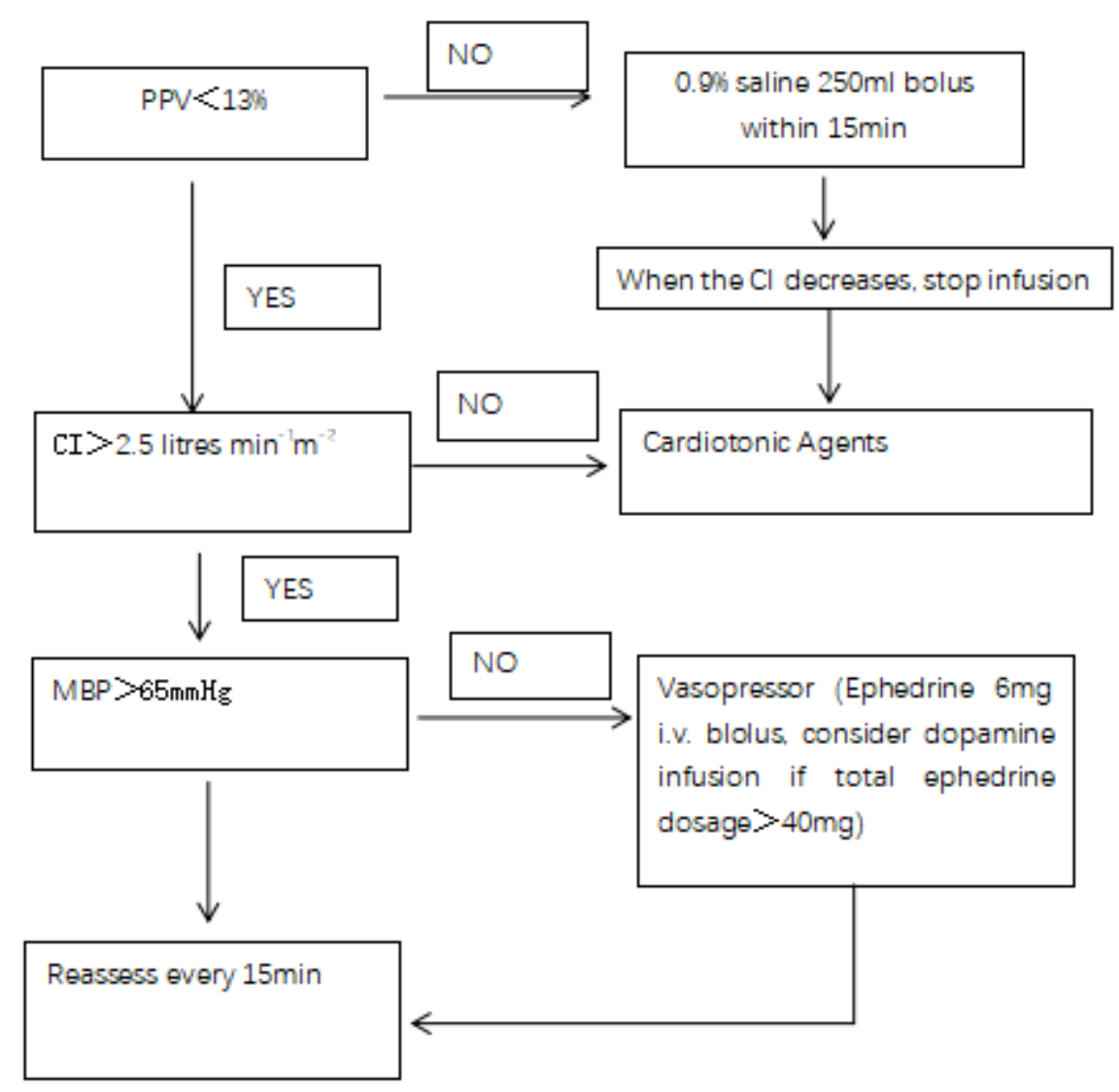


Figure 1

Protocols for PPV goal-directed fluid therapy. PPV, pulse-pressure variation; MAP, mean arterial pressure; $\mathrm{Cl}$, cardiac index.

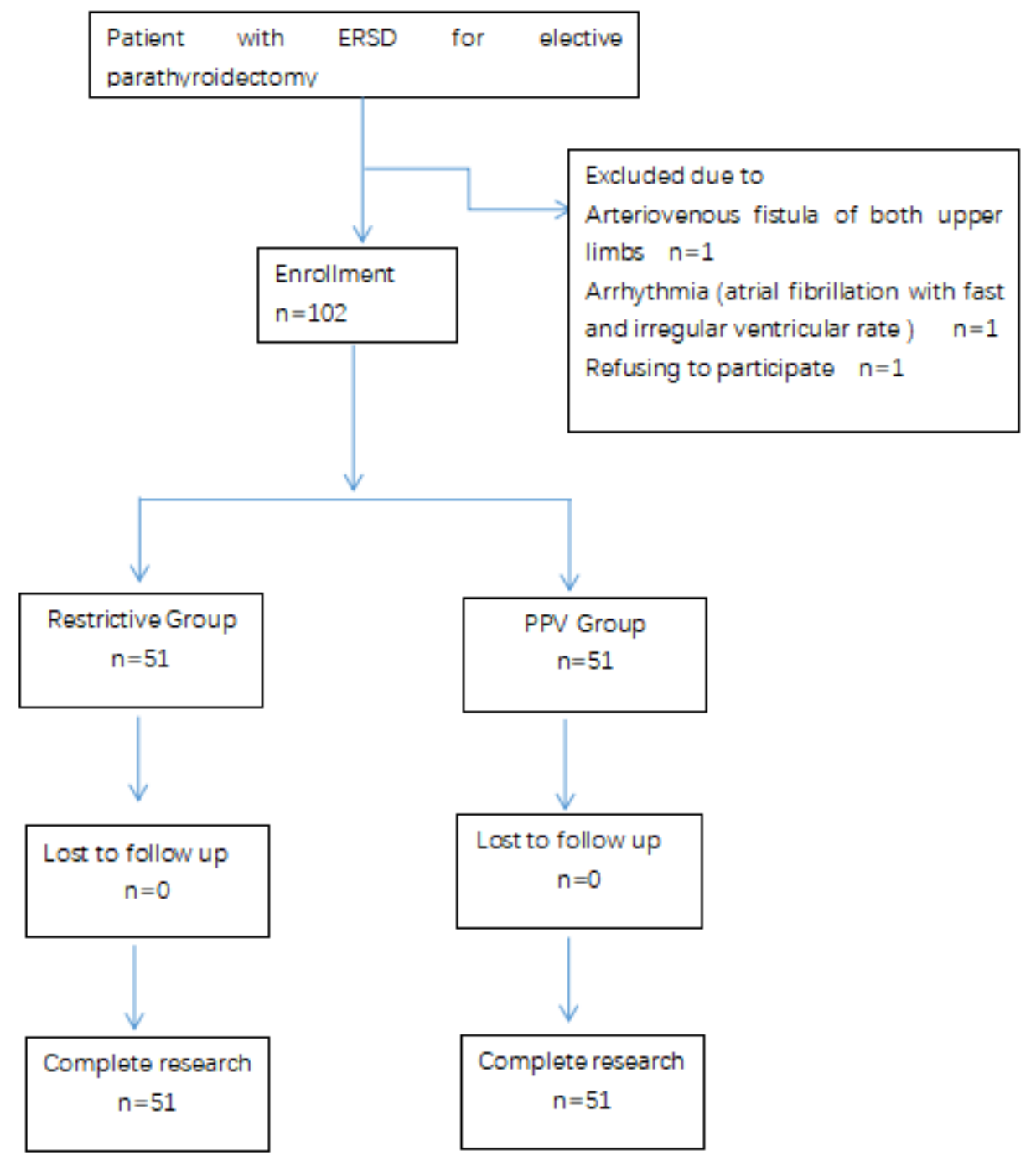

Figure 2

Patient recruitment flow chart. 

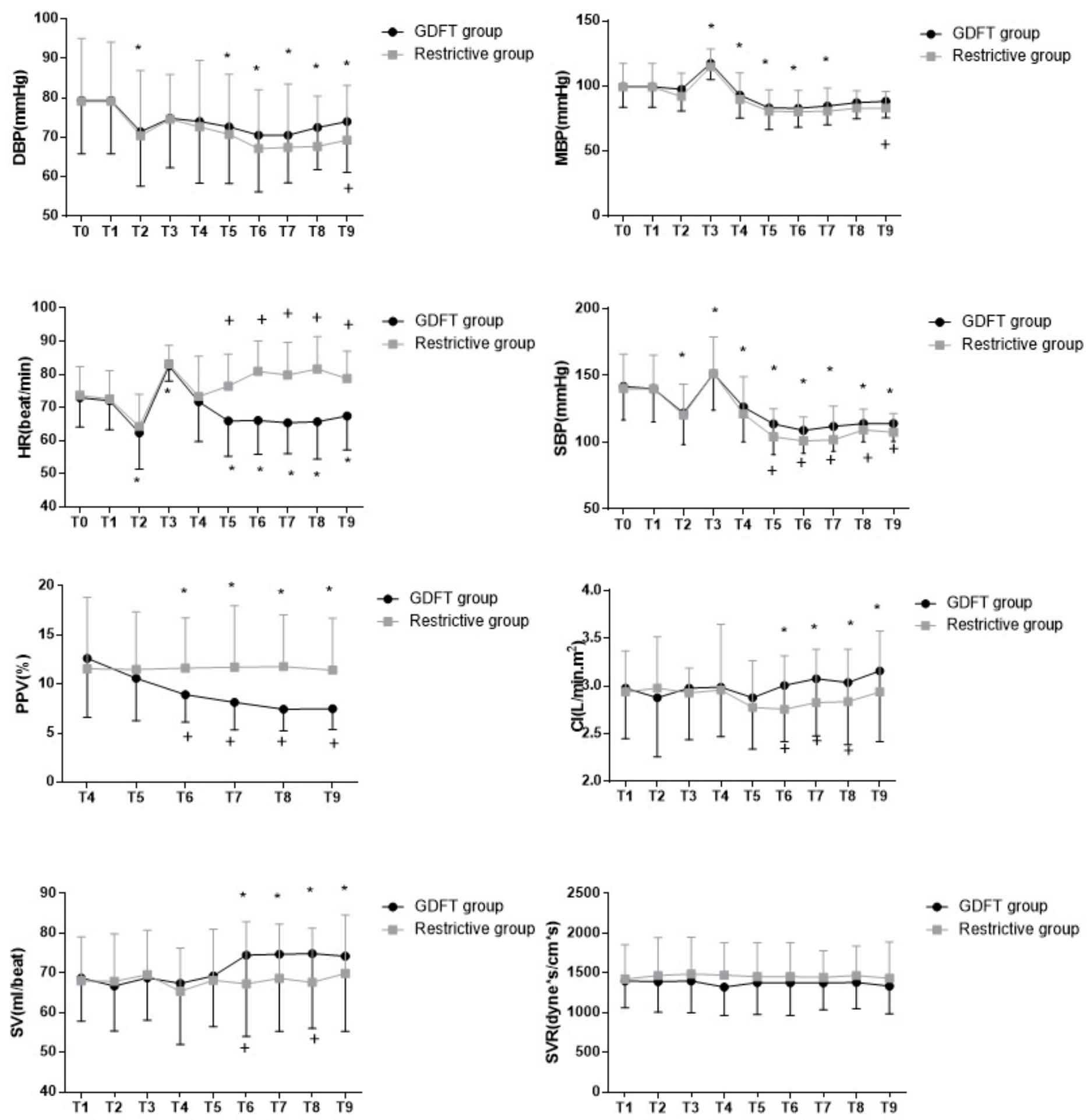

\section{Figure 3}

Differences in hemodynamic variables between the two groups at times during the perioperative period. SBP, systolic blood pressure; DBP, diastolic blood pressure; MBP, mean blood pressure; $\mathrm{HR}$, heart rate; $\mathrm{Cl}$, cardiac index; SV, stroke volume; SVR, systemic vascular resistance. T0, baseline; T1, before induction; T2, after induction; T3, immediately after intubation; T4, at the beginning of mechanical ventilation; T5, before incision; T6, $30 \mathrm{~min}, \mathrm{~T} 7,60 \mathrm{~min}, \mathrm{~T} 8,90 \mathrm{~min}$ during surgery; T9, 120 min during surgery or at the 
end of the surgery if the surgery time was less than 120 min. *Significant difference, at $P<0.05$, from baseline (T0) for SBP, DBP, MBP, and HR, and from the beginning of ventilation (T4) for PPV, Cl, SV, and SVR. + Significant difference, at $\mathrm{P}<0.05$, between the two groups (details in Supplementary Material).

\section{Supplementary Files}

This is a list of supplementary files associated with this preprint. Click to download.

- renamed1ed55.xIsx 